\title{
Planck Function Distribution in the Far Infrared Cavity nearby AGB Star at Galactic Latitude $\mathbf{0 . 6}^{\circ}$
}

\section{A. K. Gautam and D. N. Chhatkuli}

Journal of Nepal Physical Society

Volume 6, Issue 2, December 2020

ISSN: 2392-473X (Print), 2738-9537 (Online)

\section{Editors:}

Dr. Binod Adhikari

Dr. Bhawani Joshi

Dr. Manoj Kumar Yadav

Dr. Krishna Rai

Dr. Rajendra Prasad Adhikari

Mr. Kiran Pudasainee

JNPS, 6 (2), 97-103 (2020)

DOI: http://doi.org/10.3126/jnphyssoc.v6i2.34864

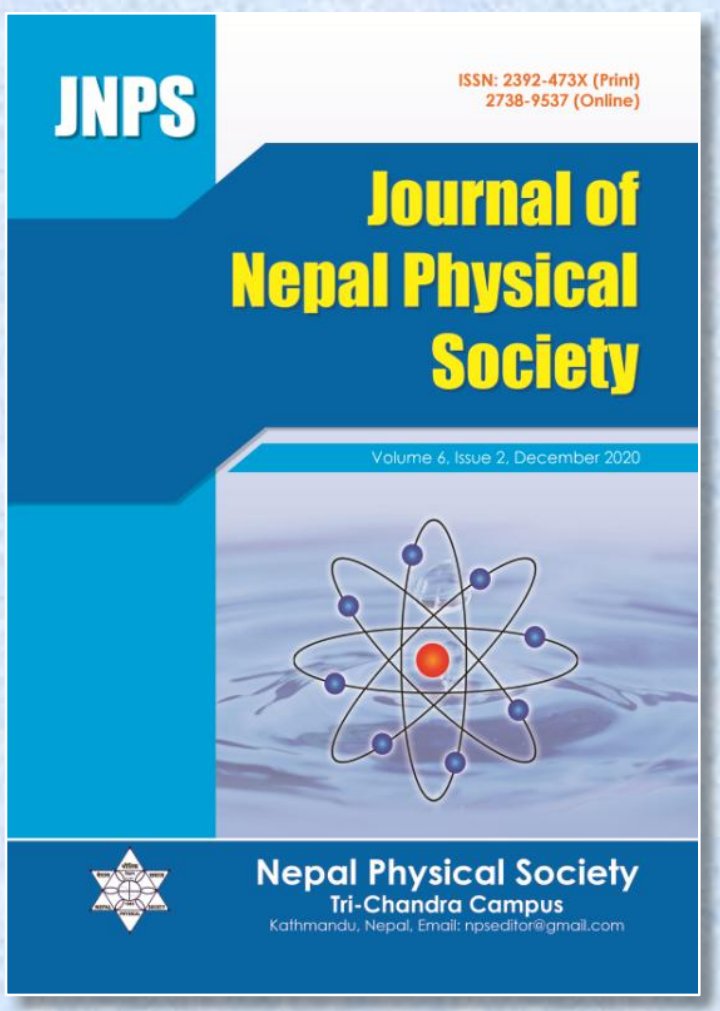

\section{Published by:}

Nepal Physical Society

P.O. Box: 2934

Tri-Chandra Campus

Kathmandu, Nepal

Email: npseditor@gmail.com 


\title{
Planck Function Distribution in the Far Infrared Cavity nearby AGB Star at Galactic Latitude $\mathbf{0 . 6}^{\circ}$
}

\author{
A. K. Gautam ${ }^{1, *}$ and D. N. Chhatkuli ${ }^{2}$ \\ ${ }^{1}$ Bhaktapur Multiple Campus, Bhaktapur, Nepal \\ ${ }^{2}$ Central Department of Physics, Kirtipur, Kathmandu, Nepal \\ *Corresponding Email: arjungautamnpj@gmail.com
}

Received: 05 October, 2020; Revised: 13 November, 2020; Accepted: 27 December, 2020

\begin{abstract}
A far infrared cavity nearby AGB star namely AGB2308+6058 was found to be located centered at R. A. $(\mathrm{J} 2000)=23^{\mathrm{h}} 06^{\mathrm{m}} 54.6^{\mathrm{s}}$ and Dec. $(\mathrm{J} 2000)=61^{\circ} 16^{\prime} 09.8^{\prime \prime}$. Distribution of dust color temperature, dust mass and visual extinction of the far infrared cavity have studied. In addition, the distribution of Planck function along the compression and extension of the cavity also has studied. This cavity has found to be located nearby the AGB star around far infrared loop G110+00 at 730 pc. It is close to the Galactic plane at galactic latitude $0.6^{\circ}$ therefore the radiation field is strong and the diameter of the cavity is found to be greater than $0.2^{0}$. The dust color temperatures was found to be in the range $22.76 \pm 0.14 \mathrm{~K}$ to $23.55 \pm 0.29 \mathrm{~K}$ with offset $0.79 \mathrm{~K}$. The contour maps of dust color temperature and dust mass showed that the low temperature region have greater mass density. Planck function showed non-uniform distribution along its extension and compression, suggesting that the dust and grains are not in the local thermodynamic equilibrium, possibly due to nearby AGB star. A clearly noted result is from counter map of dust mass and visual extinction that they showed consistent nature in their distribution.
\end{abstract}

Keywords: Dust Color Temperature, Far Infrared Cavity, Interstellar Medium, Planck Function.

\section{INTRODUCTION}

The AGB star and its surroundings are the natural laboratory in which we can study its effect in the interstellar medium. A catalog of AGB stars in IRAS PSC was developed [1]. They classified AGB stars and provided their coordinates which is the preliminary stage of our research work.

Using the extensive all sky database of IRAS, the IRAS images of nearby 100 dark molecular clouds were studied [2]. The IRAS 60 and $100 \mu \mathrm{m}$ co-added images were used to calculate dust color temperature, $100 \mu \mathrm{m}$ optical depth and visual extinction of dust in the clouds. There are 462 far infrared loops identified using 60 and $100 \mu \mathrm{m}$ processed IRAS data where they calculated distances of the 73 far infrared loops which are used to calculate dust mass around the desired region [3].

A dust structure around the planetary nebula NGC 1514 at 60 and $100 \mu \mathrm{m}$ IRAS maps were found [4]. They found the 60 and $100 \mu \mathrm{m}$ flux densities of this region and calculated dust color temperature which is around $(29 \pm 3) \mathrm{K}$. In another work, the PNNGC 2899 is located at the center of a huge quadrupolar cavity was found, whose directions of axes coincide with the directions of the main axes of the optical PN [5]. Aryal and Weinberger (2006) [6] detected a new infrared nebula, R.A. $(\mathrm{J} 2000)=08^{\mathrm{h}} 27^{\mathrm{m}}$, Dec. $(\mathrm{J} 2000)=+25^{0} 54^{\prime}$ was detected [6]. Finally, they suggested that the pulsar PSRB0823+26 might be responsible for its shaping.

The product of dust color temperature and visual extinction is consistent and their value is in the order of $10^{-4}$ mag K [7]. The contour maps of dust color temperature and dust mass showed that the low temperature region have greater mass density which supports cosmological principle. They found that the distribution of Planck function along major and minor diameters is sinusoidal, suggesting oscillation in the grain temperature distribution.

\section{DATABASE}

Infrared two-colour diagrams was presented [1] and 
found 3003 O-rich, 1168 C-rich, 362 S-type and 35 silicate carbon stars in our Galaxy. To find far infrared cavity around 1168 C-rich AGB stars at 60 and 100 $\mu \mathrm{m}$ IRIS maps using Sky View Virtual Observatory (http://skyview.gsfc.nasa.gov/current/), a systematic search was performed and selected 1 far infrared cavity for the study. The database of the far infrared (FIR) cavity is listed in Table 1. In the present work, we intend to study dust color temperature, dust mass and the distribution of Planck function along the compression and extension of the cavity.

Table 1: The database of the far infrared cavity nearby AGB star. The first column represents name (FICHH+DD), the second and third columns give positions. The last two columns list the major (a) and minor (b) diameters of the cavity and the last column represents the name of the nearby AGB star.

\begin{tabular}{|l|l|l|l|l|l|}
\hline FIC & $\alpha(\mathrm{J} 2000)(\mathrm{deg})$ & $\delta(\mathrm{J} 2000)(\mathrm{deg})$ & $\mathrm{a}(\mathrm{deg})$ & $\mathrm{b}(\mathrm{deg})$ & nearby AGB \\
\hline FIC23+61 & $23 \mathrm{~h} 06 \mathrm{~m} 54.6 \mathrm{~s}$ & $61^{0} 16^{\prime} 09.8^{\prime \prime}$ & 0.10 & 0.23 & AGB2308+6058 \\
\hline
\end{tabular}

\section{METHOD}

The method of calculation for the dust color temperature and dust mass is as described in [8]. Here, methods for calculating optical thickness and hence visual extinction are described as in [7] by using $100 \mu \mathrm{m}$ IRIS maps.

\subsection{Dust Color Temperature and Planck Function}

The dust color temperature of the FIR cavity was calculated using 60 and $100 \mu \mathrm{m}$ IRIS flux densities. For it, the method proposed by [2] was followed and later it was improved [9, 10]. Finally the derived expression for dust color temperature $T_{d}$ is written as

$$
\begin{aligned}
\mathrm{T}_{\mathrm{d}}= & \frac{-96}{\ln \left\{\mathrm{R} \times 0.6^{(3+\beta)}\right\}}, \\
& \text { where, } \mathrm{R}=\frac{\mathrm{F}(60 \mu \mathrm{m})}{\mathrm{F}(100 \mu \mathrm{m})}
\end{aligned}
$$

Where $\beta$ is the spectral emissivity index depends on dust grain properties. $\beta=0$, for a pure blackbody, $\beta$ $\sim 1$, for the amorphous layer-lattice matter and $\beta \sim$ 2 for the metals and crystalline dielectrics which is used in our calculations.

The value of Planck function depends on the wavelength (frequency), and hence temperature. Finally it is used to calculate the dust mass. The Planck function is given by the [11],

$$
\mathrm{B}(v, \mathrm{~T})=\frac{2 \mathrm{hc}}{\lambda^{3}}\left(\frac{1}{\frac{\mathrm{hc}}{\mathrm{e}^{\lambda \mathrm{kT}}-1}}\right)
$$

where, $\mathrm{h}=$ Planck constant, $\mathrm{c}=$ velocity of light, $v$ $=$ frequency at which the emission is observed, $\mathrm{T}=$ the average dust color temperature of the region.

\subsection{Dust Mass and Visual extinction}

For the calculation of dust mass, first we need the value of flux density $\left(F_{v}\right)$ at $100 \mu \mathrm{m}$ maps and we use the expression given by [12],

$$
\mathrm{M}_{\text {dust }}=\frac{4 \mathrm{ar}}{3 \mathrm{Q}_{\mathrm{n}}}\left|\frac{\mathrm{S}_{\mathrm{n}} \mathrm{D}^{2}}{\mathrm{~B}(\mathrm{n}, \mathrm{T})}\right|
$$

where, weighted grain size (a) $=0.1 \mu \mathrm{m}$, grain density $(\rho)=3000 \mathrm{~kg} \mathrm{~m}^{-3}$, grain emissivity $\left(\mathrm{Q}_{v}\right)=$ 0.0010 (for $100 \mu \mathrm{m}$ ) [13]. So the equation (3) reduces to

$$
\mathrm{M}_{\text {dust }}=0.4\left[\frac{\mathrm{S}_{\mathrm{n}} \mathrm{D}^{2}}{\mathrm{~B}(\mathrm{n}, \mathrm{T})}\right]
$$

We use equation (4) to calculate dust mass of the cavity.

Visual extinction can be estimated by using an empirical formula [2]. According to them, we have

$\mathrm{A}_{\mathrm{V}}(\mathrm{mag})=15.078\left[1-\exp \left(-\tau_{100} / 641.3\right)\right] \ldots .(5)$

Where,

$$
\tau_{100}=\frac{F_{\lambda}(100 \mu \mathrm{m})}{B_{\lambda}(100 \mu \mathrm{m})}
$$

is optical depth at $100 \mu \mathrm{m}$ wavelength. Here $F_{\lambda}$ is flux density and $B_{\lambda}$ is Planck function at $100 \mu \mathrm{m}$ wavelength.

\section{RESULT AND DISCUSSION}

We describe the physical properties of the far infrared cavity candidate and compare it with the previously published works.

\subsection{Structure of the Cavity Candidate}

From the systematic search on at $60 \mu \mathrm{m}$ and 100 $\mu \mathrm{m}$ IRAS maps, an isolated far infrared cavity at R.A. $(\mathrm{J} 2000)=23^{\mathrm{h}} 06^{\mathrm{m}} 54.6^{\mathrm{s}}$, Dec. $(\mathrm{J} 2000)=61^{0}$ $16^{\prime} 09.8^{\prime \prime}$ was found which is located around AGB star AGB23+60. JPEG images at $60 \mu \mathrm{m}$ and 100 $\mu \mathrm{m}$ IRAS map of the cavity are shown in the Fig.1 (a) and (b) respectively. From the calculation of major and minor diameter, size of the structure of the cavity was found to be $0.10^{0} \times 0.23^{0}$. Here, the core region of the cavity have minimum flux at $100 \mu \mathrm{m}$ IRIS maps, the major diameter $>0.2^{0}$ 
means it lies on the galactic plane and there is no diffuse optical emission. When it is compared with
[8], it can be suggested that cavity formed by AGB stars are quite very small than formed by pulsars.
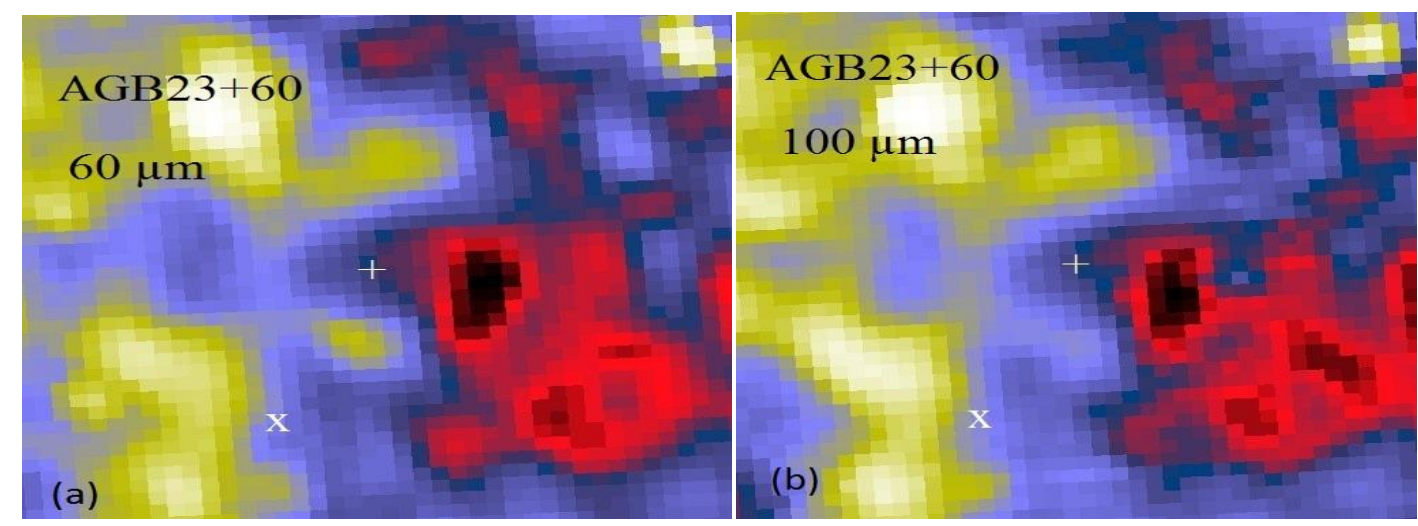

Fig. 1: (a) and (b) are the JPEG images of the far infrared cavity at $60 \mu \mathrm{m}$ and $100 \mu \mathrm{m}$ IRAS maps around the AGB $23+60$ centered at R.A. $(J 2000)=23^{\mathrm{h}} 06^{\mathrm{m}} 54.6^{\mathrm{s}}$, Dec. $(\mathrm{J} 2000)=61^{0} 16^{\prime} 09.8^{\prime \prime}$.

\subsection{Distribution of Flux Density}

By using ALADIN2.5 software, flux densities at 60 $\mu \mathrm{m}$ and $100 \mu \mathrm{m}$ have been measured. Distribution of flux density within the contour of the region of interest has studied. A graph is plotted between $F(100)$ and $F(60)$ which is shown in Fig.2(a). From the linear fit, slope of the line was 0.17 , correlation coefficient $(\mathrm{R})=0.73$. The linear equation of the fitted line is, $\mathrm{F}(60)=4.47+0.17 \mathrm{~F}(100)$. Using the slope of best fitted plot, average dust color temperature is calculated as $22.14 \mathrm{~K}$ which is used to calculate error in calculated dust color temperature.

Again distribution of flux density at $100 \mu \mathrm{m}$ of the pixels within the contour level with right ascension (R.A.) and declination (Dec.) are plotted and shown in Fig.2(b). Graph shows that all the fluxes from minimum to maximum lie within the contour level. Maximum flux regions lie at the north-east part of the contour.
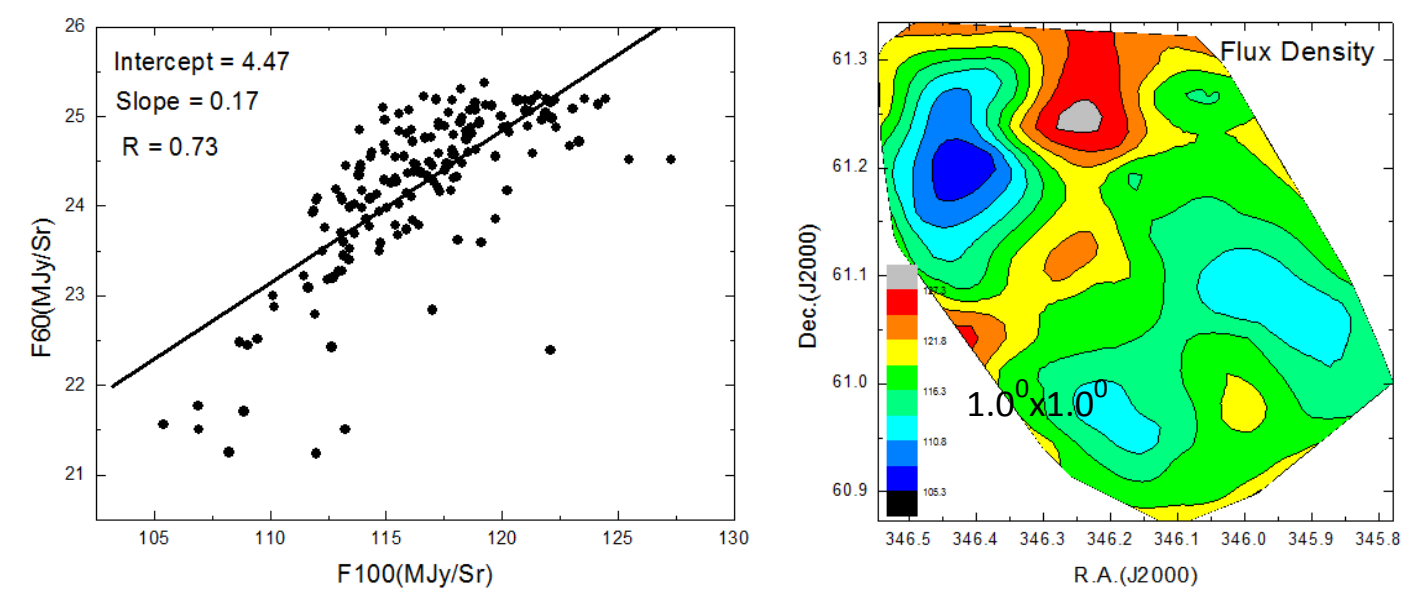

Fig. 2: The $100 \mu \mathrm{m}$ verses $60 \mu \mathrm{m}$ flux density in the region of interest (left) and Contour map at $100 \mu \mathrm{m}$ flux density (right) where the AGB star is located at the center R.A. (J2000) $=23^{\mathrm{h}} 06^{\mathrm{m}} 54.6^{\mathrm{s}}$, Dec. (J2000) $=61^{0} 16^{\prime} 09.8^{\prime \prime}$.

\subsection{Dust color Temperature and its Variation}

Dust color temperature of each pixel in the region of interest has been calculated. We use the IRAS $100 \mu \mathrm{m}$ and $60 \mu \mathrm{m}$ FITS images downloaded from the IRAS server. For the calculation of temperature, we choose the value of $\beta=2$ following the explanation given by [10]. Variation of temperature with corresponding R.A.(J2000) and Dec.(J2000) are plotted by using ORIGIN 8.0 and the graph is shown in Fig. 3(a). Graph shows that temperature distributions are in separate cluster but minimum temperature region 
is little bit shifted from minimum flux density which is unusual behaviour. Such type of nature

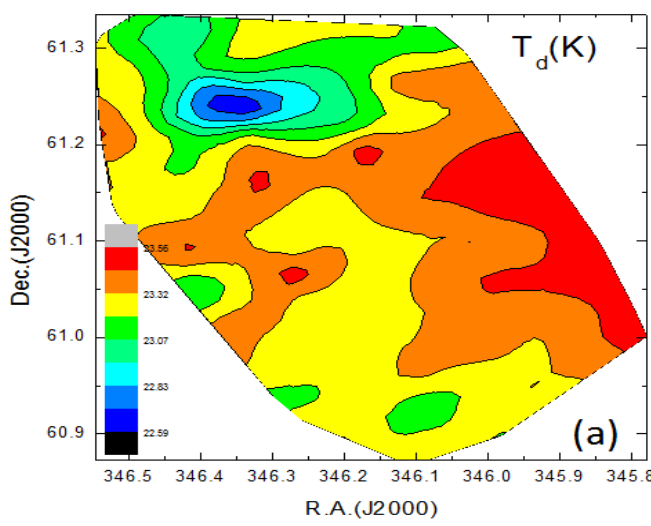

is obtained due to high mass loss by external factors.

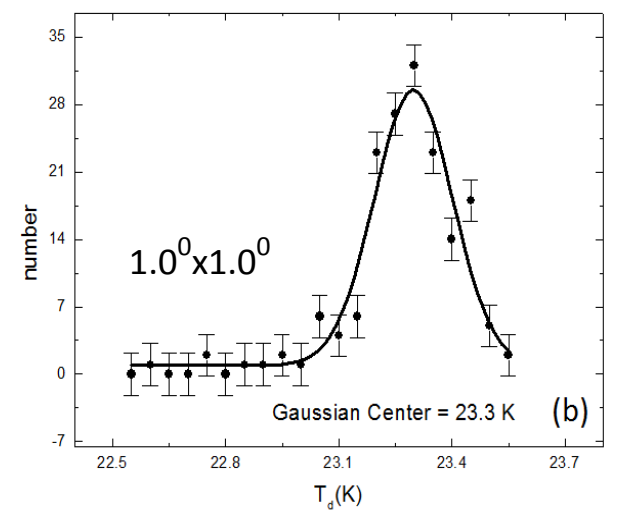

Fig. 3: (a) Contour map of dust color temperature and (b) Gaussian fit of dust color temperature. The far infrared cavity is centered at R.A. $(J 2000)=23^{\mathrm{h}} 06^{\mathrm{m}} 54.6^{\mathrm{s}}$, Dec. $(J 2000)=61^{0} 16^{\prime} 09.8^{\prime \prime}$.

The minimum and maximum temperature region was found in the range $(22.76 \pm 0.14) \mathrm{K}$ to $(23.55 \pm 0.29)$ $\mathrm{K}$ respectively with offset value $0.81 \mathrm{~K}$. Such low offset temperature variation shows that there is symmetric outflow or symmetric distribution of density and temperature. It further suggests that particles are independently vibrating. Gaussian fit of dust color temperature is more or less following the Gaussian distribution with positive skewness. When this result is compared with the result obtained in [14] where temperature variation is $20 \mathrm{~K}$ to $22 \mathrm{~K}$ so our result is also comparable with that result. In the contour map, minimum flux and minimum temperature region are shifted which is due to some external factors possibly due to AGB wind.

Dust color temperature of two far infrared cavities

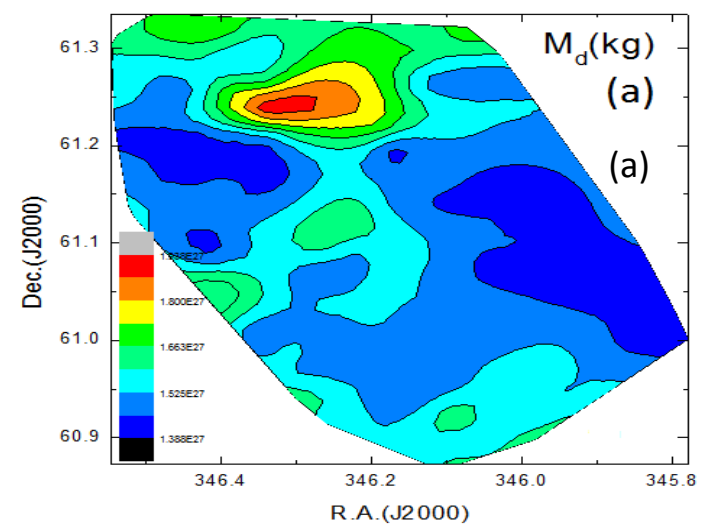

lying nearly at the galactic plane $\left(-3^{0}\right)$ using IRAS maps was measured [15]. They measured dust color temperature of the two cavities which was in the range $23.4 \mathrm{~K}$ to $24.1 \mathrm{~K}$ with an offset of only $0.7 \mathrm{~K}$ and $22.2 \mathrm{~K}$ to $24.6 \mathrm{~K}$, with an offset of about $2.4 \mathrm{~K}$ respectively. Range of this result is similar but offset is different with our result.

\subsection{Dust Mass Estimation and its variation}

Since the distance of the structure is $730 \mathrm{pc}$ [3]. So, by using the temperature of each pixel and corresponding distance of the structure, we calculated mass of each pixel. Total mass of the structure is $2.57 \times 1029 \mathrm{~kg}$ i.e $0.129 \mathrm{M}_{\odot}$. But mass of dust obtained around white dwarf WD $1003-44$ in [14] is $0.08 \mathrm{M}_{\odot}$. It means mass of dust around AGB Star is less than White Dwarf.

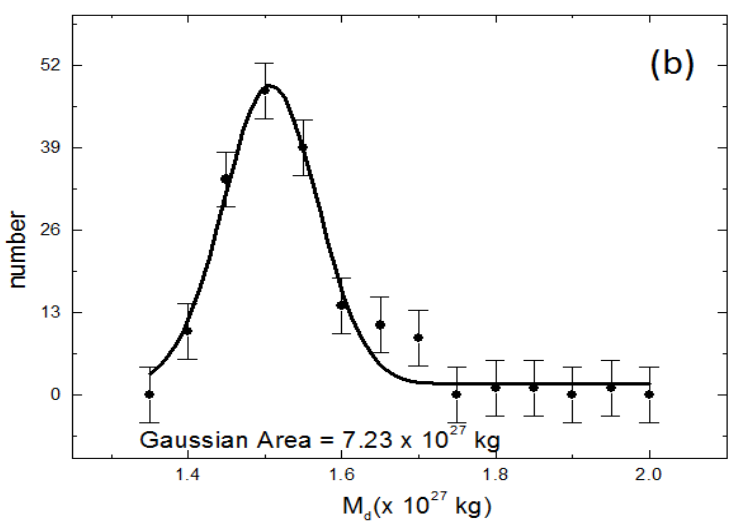

Fig. 4: (a) contour map of dust mass and (b) Gaussian fit of dust mass. The far infrared cavity is centered at R.A. $(J 2000)=23^{\mathrm{h}} 06^{\mathrm{m}} 54.6^{\mathrm{s}}$, Dec. $(\mathrm{J} 2000)=61^{0} 16^{\prime} 09.8^{\prime \prime}$.

Distribution of dust mass in the contour map is shown in figure 4(a) which shows that minimum temperature region is denser and lie at the maximum mass region in the selected contour which is usual trend and. It means 
distribution of dust mass follow cosmological principle i.e their distribution is homogeneous and isotropy. Figure 4(b) is the Gaussian fit where the data more or less are Gaussian with positive skewness. Total dust mass of the cavity nearby planetary nebula was found to be $3.5 \mathrm{M}_{\odot}[16]$ which too much greater than our far infrared cavity nearby AGB star. From these two results, It can be suggested that FIR cavity around AGB star are either in small in size or contain carbon or silicon dust particles.

\subsection{Distribution of Planck function with Diameters}

Distribution of Planck function along extension and compression are shown in Fig. 5(a) and (b) where the data are distributed randomly with very low correlation coefficient $(\mathrm{R})=0.91$ in case of major diameter but in case of minor diameter, $\mathrm{R}=-0.97$. There is no systematic trend of their distribution in both cases. It means distribution is non uniform and is not following the Maxwell velocity distribution which is possibly due to nearby AGB wind. Variation of Planck function with diameters and found uniform distribution in case of pulsars was studied [8] but in our case i.e., in case of AGB stars, non-uniform distribution was found. It justifies that pulsars emit radiation and the AGB stars emit material in high speed.
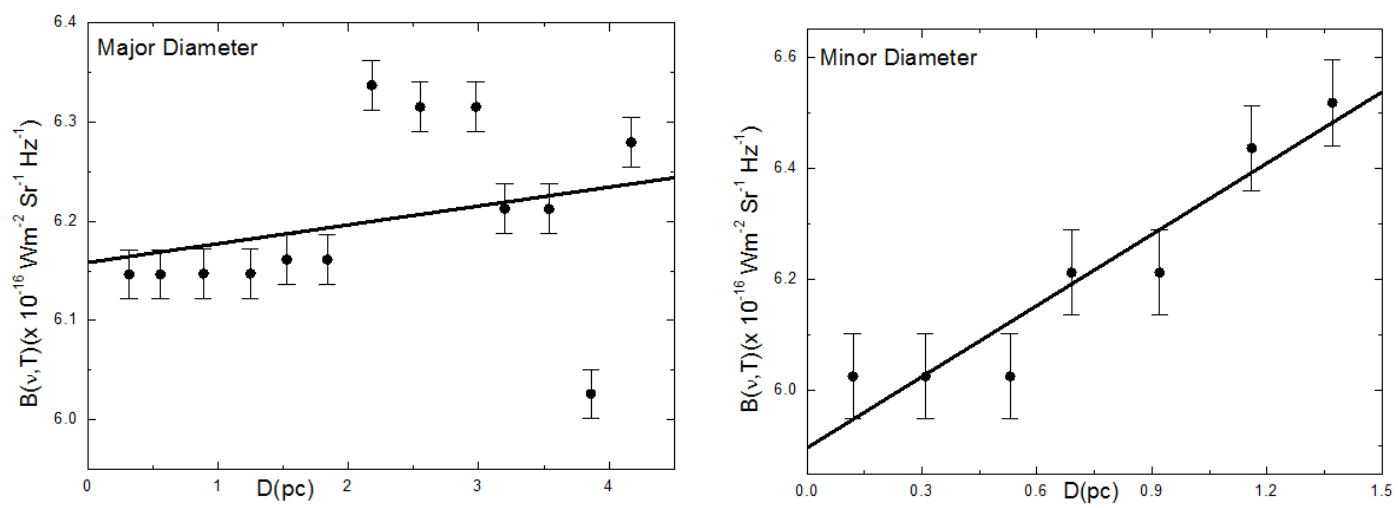

Fig. 5: (a) Linear fit of scattered plot between major diameter and Planck function and (b) Linear fit of scattered plot between minor diameter and Planck function of the cavity centered at R.A. $(J 2000)=23^{\mathrm{h}} 06^{\mathrm{m}} 54 \cdot 6^{\mathrm{s}}$, Dec. $(J 2000)=61^{0} 16^{\prime} 09.8^{\prime \prime}$.

\subsection{Variation of Visual Extinction with Dust Color Temperature}

Linear fit of the scattered plot between visual extinction and dust color temperature is shown in Fig. 6(a). The graph shows a systematic trend with high correlation coefficient i.e., -0.82 , which shows that there is best correlation between the data. From the linear fit, we found a new relation between visual extinction and dust color temperature. The relation between visual extinction and dust color temperature is $\left(A_{V} \times T_{d}\right)=4 \times 10^{-4}$ which also lies in the range proposed by [7]. Figure 6(b) is the contour map of visual extinction. When it is compared with the contour map of dust color temperature, it is found that higher the dust color temperature, lower the visual extinction and vice-versa. Similar nature was obtained in case of AGB stars $[\mathbf{1 7}, \mathbf{1 8}]$ and in case of post-AGB stars [19, 20].
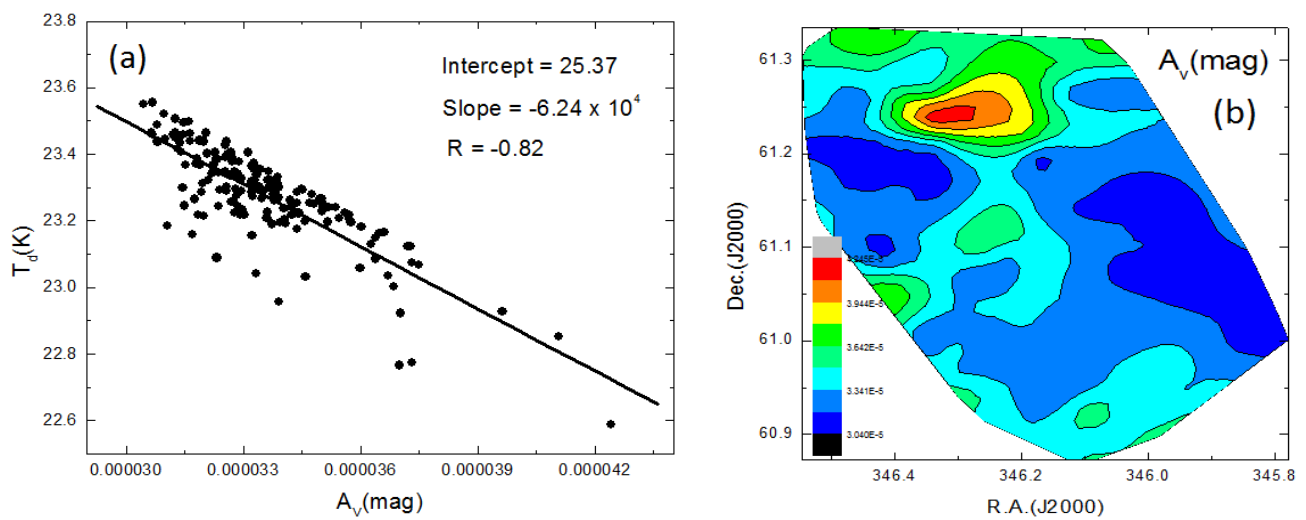

Fig. 6: (a) A linear fit of the scattered plot between visual extinction and dust color temperature and (b) contour map of visual extinction of the far infrared cavity centered at R.A. $(J 2000)=23^{\mathrm{h}} 06^{\mathrm{m}} 54.6^{\mathrm{s}}$, Dec. $(J 2000)=61^{0} 16^{\prime} 09.8^{\prime \prime}$. 


\section{CONCLUSION}

The physical properties of the cavity at far infrared $(60 \mu \mathrm{m}$ and $100 \mu \mathrm{m})$ IRIS maps, located nearby AGB stars are presented. The dust color temperature, dust mass, visual extinction and Planck function distribution along the diameters are studied. We conclude our results as follows:

1. Minimum and maximum dust color temperature were found to be $(22.76 \pm 0.14) \mathrm{K}$ and $(23.55 \pm$ $0.29) \mathrm{K}$ respectively with offset value $0.81 \mathrm{~K}$. Such low off set value suggests that the cavity might be in thermodynamic equilibrium. The dust color maps compliment the dust mass maps in the FIR cavity. The minimum temperature region was found to be massive supporting the cosmological principle.

2. A very good correlation was noticed between the visual extinction and dust color temperature. The product of dust color temperature and visual extinction was found to be $\left(4 \times 10^{-4}\right) \mathrm{K}$ mag which is very less than 1 . Contour map of dust color temperature and visual extinction showed that higher the dust color temperature, lower the visual extinction and vice-versa.

3. A fluctuation in the distribution of Planck function along both diameters of the FIR cavity was noticed, suggesting that the dust particles are oscillating non-uniformly.

4. Total mass of the structure is $2.57 \times 10^{29} \mathrm{~kg}$ i.e $0.129 \mathrm{M}_{\odot}$. The contour maps showed that the low temperature region have greater mass density. Distribution of dust mass is supporting cosmological principle.

This work will be intended to study the cavities in different bands using IRAS, AKARI and WISE survey in the future.

\section{ACKNOWLEDGEMENTS}

Author is gratefulto Prof. Dr. Binil Aryal, $\mathrm{HoD}$ of central department of physics and Prof. R. Weinberger, Department of Astro-Particle Physics, Innsbrck University, for invoking me to work on dusty environments around AGB stars. This research has made use of SkyView Virtual Observatory, Aladin v2.5 and NASA/IPAC Extragalactic Database (NED).

\section{REFERENCES}

[1] Suh, K. W.; \& Kwon, Y. J. A Catalog of AGB Stars in IRAS PSC. Journal of Korean Astronomical Society, 42: 81-91 (2009).
[2] Wood, D. O. S.; Myers, P. C.; \& Daugherty, D. A. IRAS images of nearby dark clouds. The Astrophysical Journal Supplement, 95: 457-501 (1994).

(3) Könyves, V.; Kiss, C.; Moór, A.; Kiss, Z. T.; \& Tóth, L. V. Catalogue of far-infrared loops in the Galaxy. Astronomy and Astrophysics, 463: 12271234 (2007).

[4] Aryal, B.; Rajbahak, C.; \& Weinberger, R. A giant dusty bipolar structure around the planetary nebula ngc 1514. Monthly Notices of the Royal Astronomical Society, 402: 1307-1312 (2010).

[5] Aryal, B.; Rajbahak, C.; Weinberger, R. Planetary nebulae NGC 6826 and NGC 2899: early aspherical mass loss? Astrophysics and Space Science, 323 (4): 323-327(2009).

[6] Aryal, B.; \& Weinberger, R. A new large high latitude cone-like far-ir nebula. Astronomy and Astrophysics, 448: 213-219 (2006).

[7] Gautam, A. K.; \& Aryal, B. A study of lowlatitude (I I $<10^{\circ}$ ) far infrared cavities. Journal of Astrophysics and Astronomy, 40 (16): 1-10 (2019).

[8] Jha, A.; Aryal, B.; \& Weinberger, R. A study of dust color temperature and dust mass distributions of four far infrared loops. Revista Mexicana deAstronomíay Astrofísica, 53(2): 467-476 (2017).

[9] Dupac, X.; Bernard, J. P.; Boudet, N.; Giard, M.; Lamarre, J. M.; Meny, C.; Pajot, F.; Ristorcelli, I.; Serra, G.; Stepnik, B. \& Torre, J. P. Inverse Temperature Dependance of the Dust Submillimeter Spectral Index. Astronomy \& Astrophysics, 404: L11-L15 (2003).

[10] Schnee, S. L.; Ridge, N. A.; Goodman, A. A. \& Jason, G. L. A Complete Look at the Use of IRAS Emission Maps to Estimate Extinction and Dust Temperature. The Astrophysical Journal, 634: 442-450 (2005).

[11] Beichman, C. A.; Wilson, R. W.; Langer, W. D.; \& Goldsmith, P. F. Infrared limb brightening in the Barnard 5 cloud. The Astrophysical Journal Letters, 332: L81-L85 (1988).

[12] Hildebrand, R. H. The determination of cloud mass and dust characteristics from sub millimeter thermal emission. Royal Astronomical Society, 24: 267-282 (1983).

[13] Young, K.; Phillips, T. G. \& Knapp, G. R. Circumstellar Shells Resolved in IRAS Survey Data II. Analysis. Astrophysical Journal, 409: 725-738 (1993).

[14] Aryal, B. \& Weinberger, R. Dust structure around White Dwarf WD 1003-44 in 60 and $100 \mu \mathrm{m}$ Iras Survey. The Himalayan Physics, 2: 5-10 (2011).

[15] Jha, A. K. \& Aryal, B. Dust color temperature distribution of two fir cavities at iris and akari 
maps. Journal of Astrophysics and Astronomy, 39: 24-32 (2018).

[16] Aryal, B. \& Weinberger, R.. Temperature and Mass Profile of Far-infrared Skeleton Nebula. The Himalayan Physics, 1: 1-3 (2010).

[17] Gautam, A. K. \& Aryal, B. Study of two Far Infrared cavities Nearby Asymptotic Giant Branch Star Under Infrared Astronomical Satellite Maps. Journal of Institute of Science and Technology, 24(2): 76-84 (2019).

[18] Gautam, A. K. \& Aryal, B. Study of dust color temperature and visual extinction distribution of a far infrared cavity at 60 and $100 \mu \mathrm{m}$ IRAS map around the AGB star at galactic latitude $8.6^{\circ}$. BIBECHANA, 17: 42-49 (2020).

[19] Gautam, A. K. \& Aryal, B. Far infrared cavity of a post C-rich AGB star under IRAS survey. BIBECHANA, 15: 90-96 (2018).

[20] Gautam, A. K. \& Aryal, B. Study of Far Infrared Cavity Around a Post-AGB Star Under IRAS Survey at Galactic Latitude $0.3^{0}$. BIBECHANA, 16: 23-30 (2019). 